\title{
Eruptions of the V838 Mon type: stellar merger versus nuclear outburst models
}

\author{
R. Tylenda ${ }^{1}$ and N. Soker ${ }^{1,2}$ \\ 1 Department for Astrophysics, N. Copernicus Astronomical Center, Rabiańska 8, 87-100 Toruń, Poland \\ e-mail: tylenda@ncac.torun.pl \\ 2 Department of Physics, Technion-Israel Institute of Technology, 32000 Haifa, Israel \\ e-mail: soker@physics.technion.ac.il
}

Received 14 September 2005 / Accepted 6 January 2006

\begin{abstract}
We discuss various models and scenarios proposed to explain the nature of the V838 Mon type eruptions. In this class of eruptive objects we include: M31 RV (erupted in 1988), V4332 Sgr (erupted in 1994) and V838 Mon (erupted in 2002). We concentrate on three models: (i) thermonuclear runaway on an accreting white dwarf (nova-like event); (ii) He-shell flash in a post asymptotic giant branch star (born-again AGB); and (iii) merger of stars. We show that models (i) and (ii) cannot account for the majority of the observed properties of the objects. Most significantly, in both nuclear burning type models the object is expected to heat up before declining and fade as a very hot compact star. In the observed eruptions the objects declined as very cool giants or supergiants. We show that the stellar merger model can account for all the observed properties and conclude that presently this is the most promising model to explain the eruptions of the V838 Mon type.
\end{abstract}

Key words. stars: supergiants - stars: binaries: general - stars: pre-main sequence - stars: mass loss - stars: variables: general - stars: general

\section{Introduction}

The outburst of V838 Mon, discovered at the beginning of January 2002 (Brown 2002), was an important event in the field of stellar eruptions. Initially thought to be a nova, the object soon appeared unusual and enigmatic in its behaviour. The eruption, as observed in the optical, lasted about three months. After developing an $\mathrm{A}-\mathrm{F}$ supergiant spectrum at the optical maximum at the beginning of February 2002, the object showed a general tendency to evolve to lower effective temperatures. In April 2002 it almost disappeared from the optical but remained very bright in infrared, becoming one of the coolest M-type supergiants yet observed.

The interest raised by V838 Mon recalled two similarly behaving objects observed in the past. These were M 31 RV, a red variable discovered in M 31 in September 1988 (Rich et al. 1989), and V4332 Sgr, discovered at the end of February 1994 (Hayashi et al. 1994). Forgotten for almost 10 years V4332 Sgr appeared, with new observations done in 2003, to show very unique spectral features: a strong emission-line spectrum of very low excitation and a huge infrared excess.

We call M 31 RV, V4332 Sgr and V838 Mon V838 Mon type objects. Their nature is unclear. As mentioned in a number of papers, thermonuclear models (classical nova, He-shell flash) seem to be unable to explain these eruptions. This view is, however, not a consensus opinion and thermonuclear models are still discussed in the literature. Munari et al. (2005) propose a thermonuclear shell event in an evolved massive star approaching carbon ignition, while Lawlor (2005) considers a very late He-shell flash in a post-AGB (post asymptotic gaint branch) star with a period of accretion added during the flash. Other proposed explanations invoke stellar mergers (Soker \& Tylenda 2003) and giants swallowing planets (Retter \& Marom 2003).

In the present paper we make a comprehensive comparison between various models and scenarios proposed to explain the nature of the V838 Mon type eruptions and the observations of these objects. For this purpose, in Sect. 2, we summarize principle observational properties of the objects. In Sect. 3, we discuss thermonuclear eruption models and we show that they cannot consistently explain the observations. In Sect. 4 we develop the stellar merger model proposed in Soker \& Tylenda (2003) and show that it can satisfactorily account for all the observed properties of the V838 Mon type objects. A summary is given in Sect. 5 .

\section{Observed properties}

The principal observational property that makes M31 RV, V4332 Sgr and V838 Mon form a separate class of eruptive 
objects is their spectral and photometric evolution in late phases of eruption, in particular during decline. The evolution during outburst has been observed only in V838 Mon, so we do not know if its light curve properties, i.e. multi-outburst character (Crause et al. 2003; Retter \& Marom 2003), are also characteristic of the other two objects. Both M 31 RV and V4332 Sgr very likely had been discovered at a late phase of eruption. Their observed evolution was very similar to that of V838 Mon starting in last days of March 2002, i.e. about 3 months after its discovery. In this phase the objects resambled K-type or early M-type supergiants and on a time scale of days they evolved to later spectral types (Mould et al. 1990; Martini et al. 1999; Tylenda 2005). Soon they became the coolest known supergiants or giants. The latest recorded spectrum of M31 RV was M6I, corresponding to $T_{\text {eff }} \simeq 2600 \mathrm{~K}$ (Mould et al. 1990), but after, judging from the subsequent infrared photometry, the object evolved to even lower effective temperatures. V4332 Sgr reached a spectral type as late as M 8-9 III and a corresponding effective temperature of $\sim 2300 \mathrm{~K}$ (Martini et al. 1999), although it is not excluded that the object dropped to even lower $T_{\text {eff }}$. In the coolest phase of V838 Mon its spectral type was usually being refered to as later than M 10 (Desidera \& Munari 2002) while its effective temperature was as low as $2000 \mathrm{~K}$ (Tylenda 2005).

Before reaching early $\mathrm{M}$-type spectral classes, i.e. for $T_{\text {eff }} \gtrsim$ $3600 \mathrm{~K}$, the objects evolved at a roughly constant luminosity. Near the spectral type M0 all the three objects began to decline. Initially the luminosity decrease was rather fast and a factor of 10 in the luminosity drop was reached after $\sim 50, \sim 10$ and 50-100 days in the case of M 31 RV (Mould et al. 1990), V4332 Sgr (Tylenda et al. 2005a) and V838 Mon (Tylenda 2005), respectively. At this luminosity level V838 Mon and presumably also V4332 Sgr were close to their minimum effective temperatures. All that is known about the subsequent evolution of M $31 \mathrm{RV}$ is that it became fainter by a factor of 100 after 300 days (Mould et al. 1990). V838 Mon, after having reached its coolest state, kept declining in luminosity but at a much slower rate and with a slowly increasing effective temperature. About 1000 days after eruption the object was $\sim 40$ times fainter than at maximum, with $T_{\text {eff }} \simeq 2600 \mathrm{~K}$ corresponding to a spectral type of $\sim \mathrm{M} 6$ (Tylenda 2005). At 23500 days after eruption V4332 Sgr decreased by a factor of $\sim 1500$ in luminosity, its spectral type was then $\sim \mathrm{M} 3$ and $T_{\text {eff }} \simeq 3400 \mathrm{~K}$ (Tylenda et al. 2005a). For the three objects there were no indications of any excursion to high effective temperatures during decline and no nebular stage was observed. The objects declined at very low effective temperatures.

The maximum luminosity attained by an eruptive star is an important observational parameter in understanding the nature of the object. Unfortunately it depends on the distance which is usually uncertain for Galactic objects. Therefore M31 RV is the only object for which we can be confident of its maximum luminosity of $\sim 8 \times 10^{5} L_{\odot}$ (Mould et al. 1990). For a distance to V838 Mon of $8 \mathrm{kpc}$ the maximum luminosity of this object was $\sim 1.2 \times 10^{6} L_{\odot}$ (Tylenda 2005). However, because of distance uncertainties this value is probably uncertain by a factor of $\sim 2$. Even more uncertain is the maximum luminosity of V4332 Sgr. If its progenitor had been a main sequence star the object would have attained $\sim 5 \times 10^{3} L_{\odot}$ (Tylenda et al. 2005a), which would be significantly lower than in the two other cases.

The outburst amplitude is independent of distance so, in principle, it can be determined more precisely than the luminosity. Unfortunately only an upper limit is available for the brightness of M $31 \mathrm{RV}$ before outburst so it can only be said that the object brightened by at least $\sim 5 \mathrm{mag}$ in the $I$ band (Mould et al. 1990). More data are available for the progenitors of V4332 Sgr and V838 Mon so it can be estimated that at maximum these objects were, respectively, $\sim 5 \times 10^{3}$ and $(0.5-1.5) \times 10^{3}$ times more luminous than before the eruption (Tylenda et al. 2005a,b; Tylenda 2005).

The three objects probably lost significant mass. However, direct evidence, i.e. P-Cygni line profiles, have been observed only for V838 Mon. They give wind velocities up to $\sim 600 \mathrm{~km} \mathrm{~s}^{-1}$ although most of mass loss occured at $150-400 \mathrm{~km} \mathrm{~s}^{-1}$ (Munari et al. 2002b; Kolev et al. 2002; Crause et al. 2003; Kipper et al. 2004). Similar values, i.e. $270-400 \mathrm{~km} \mathrm{~s}^{-1}$, result from the observed expansion of the effective photosphere in eruption (Tylenda 2005). Widths of emission lines observed in V4332 Sgr, if interpreted as primarily due to expansion, indicate a velocity of $\sim 100 \mathrm{~km} \mathrm{~s}^{-1}$ (Martini et al. 1999). Expansion velocities of a few hundreds $\mathrm{km} \mathrm{s}^{-1}$ have been estimated for M31 RV (Mould et al. 1990; Iben \& Tutukov 1992).

The total mass lost in eruption is uncertain for the objects. Very rough estimates done for M31 RV give values between $10^{-3}-10^{-1} M_{\odot}$ (Mould et al. 1990). No estimate has been done for V4332 Sgr. Rushton et al. (2003) obtained an upper limit of $\sim 0.01 M_{\odot}$ for the total mass lost from V838 Mon from their $\mathrm{CO}$ and $\mathrm{SiO}$ observations. This upper limit should, however, be multiplied by at least a factor of 10. Rushton et al. have integrated their upper limits for the line fluxes over a velocity interval of $50 \mathrm{~km} \mathrm{~s}^{-1}$, which is much too low compared to the observed expansion velocities (see above). Also, their estimates assume that all the ejecta were seen in the lines (optically thin limit). Tylenda (2005) concludes that the total mass lost by V838 Mon is between $\sim 5 \times 10^{-3} M_{\odot}$ and $\sim 0.6 M_{\odot}$. Lynch et al. (2004) give $\sim 0.04 M_{\odot}$ with a large uncertainty. An integration of the luminosity derived by Tylenda (2005) over the pre-eruption and eruption phase gives a value of $\sim 2.5 \times 10^{46} \mathrm{erg}$. Assuming equipartition of energy between radiation and mass loss, as well as a wind velocity of $300 \mathrm{~km} \mathrm{~s}^{-1}$ one obtains mass loss of $\sim 0.03 M_{\odot}$.

In the case of V4332 Sgr and V838 Mon inverse P-Cygni profiles were observed during the decline, indicating an infall velocity of $\sim 20 \mathrm{~km} \mathrm{~s}^{-1}$ (Martini et al. 1999; Rushton et al. 2005a).

The three objects in decline show strong bands from molecules involving oxygen, clearly indicating oxygen-rich $(\mathrm{C} / \mathrm{O}<1)$ matter. More detailed determinations of the chemical composition are available only for V838 Mon (Kipper et al. 2004; Kaminsky \& Pavlenko 2005). The abundances of the iron group metals are slightly below the solar values, $[\mathrm{Fe} / \mathrm{H}] \simeq-0.3$. Those of $\mathrm{Li}, \mathrm{Ba}$ and $\mathrm{La}$ are somewhat above, although as noted in Kipper et al. (2004), the obtained abundances of these elements are sensitive to the (uncertain) microturbulence velocity. Thus it can be concluded that the observed 
abundances in V838 Mon are consistent with unprocessed matter at the galactocentric distance of the object.

Nothing is known about the progenitor of M $31 \mathrm{RV}$. In the case of V4332 Sgr, archival photometric data suggest an F-K type star (Tylenda et al. 2005a; Kimeswenger 2005). V838 Mon has a B3V spectroscopic companion (Munari et al. 2002a). The companion is very likely to be related to V838 Mon itself, e.g. forming a binary system, although a pure coincidence in the sky cannot be excluded. If it is a binary, V838 Mon before the outburst was a star similar to its B-type companion, i.e. an early B-type main-sequence star or an A-type pre-main-sequence star (Tylenda et al. 2005b). The object is surrounded by extended circumstellar matter seen in the light echo (Munari et al. 2002b; Bond et al. 2003). The matter is most likely of interstellar origin, possibly being part of one of the star forming regions seen in the direction of and at similar distances as V838 Mon (Tylenda 2004; Tylenda et al. 2005b).

Circumstellar matter is also seen in V4332 Sgr, strongly radiating in the infrared as observed $\sim 9$ years after outburst (Banerjee et al. 2003, 2004). Most likely this matter forms a circumstellar disc dissipating its energy in viscous processes (Tylenda et al. 2005a). The origin of the disc is however unclear. The object also shows an intense emission-line spectrum due to neutral elements and molecules (Banerjee \& Ashok 2004; Tylenda et al. 2005a). A possible interpretation is that this emission is due to optically thin (in the continuum) matter left after outburst and now orbiting the central star at a few stellar radii (Tylenda et al. 2005a).

V838 Mon was observed with Chandra a year after outburst (Orio et al. 2003). The object was not detected and an upper limit to the X-ray luminosity was $\sim 0.13 L_{\odot}$ for a distance of $8 \mathrm{kpc}$.

$\mathrm{SiO}$ maser emission was detected in V838 Mon $\sim 3$ years after its outburst (Deguchi et al. 2005). Its intensity increases with time (Claussen et al. 2005).

\section{Problems with thermonuclear eruption models}

A sudden brightening of a star by a factor of $\sim 10^{3}$ is usually interpreted as due to a thermonuclear eruption. This is because most astrophysical events of this range are nova-type outbursts. Their observational properties can be consistently explained by thermonuclear runaway on an accreting white dwarf. Another mechanism based on nuclear burning is a He-shell flash in an evolved star. Here we discuss the observed properties of the V838 Mon type eruptions in terms of these two thermonuclear mechanisms.

\subsection{Nova-like outbursts}

The V838 Mon type eruptions may be considered as nova-like events. The nova mechanism, thermonuclear runaway on an accreting white dwarf, has been well studied and is known to be able to account for a large variety of astrophysical outbursts. This includes relatively "gentle" eruptions lasting decades in some symbiotic stars, slow novae that require months to reach maximum and that then can stay active for years, and fast novae which in an hour or so increase their brightness by 3-4 orders of magnitude and violently eject matter with velocities well above $1000 \mathrm{~km} \mathrm{~s}^{-1}$. Indeed several objects of the nova type, like some slow novae, have light curves similar to that of V838 Mon. An example is HR Del (Nova Del 1967), which showed an initial increase in luminosity that lasted $\sim 5$ months, then two luminosity peaks with a five month separation between them, and then a decline over many years (e.g., Drechsel et al. 1977; Rafanelli \& Rosino 1978). Scaling the luminosity upward and shrinking the time scale by a factor of $\sim 5$ will lead to a light curve qualitatively similar to that of V838 Mon. Friedjung (1992) pointed out the unusual behavior of this nova in having an almost stationary photosphere. This is qualitatively similar to V838 Mon, which also seemed to have a quasistatic photosphere in its pre-outburst (January 2002) phase (Tylenda 2005). However, the light curve is probably the only observational feature of the V838 Mon type objects that can be easily interpreted in terms of a nova-like event. As already noted in early analyses of M31 RV (Mould et al. 1990) and V4332 Sgr (Martini et al. 1999), several observational properties of these objects are difficult to reconcile with the nova hypothesis.

The crucial argument against the nova-like event comes from the observed spectral evolution of the objects during outburst and decline. In the case of a classical nova event, after an outburst on a degenerate white dwarf, steady nuclear burning commences, which halts the luminosity typically at $\sim 10^{4} L_{\odot}$. It can be extinguished only after the envelope mass becomes too small to keep the burning shell dense and hot enough. But this implies that the photospheric radius must be small, hence the effective temperature high. This is the reason why novae after eruption (but also post-AGB stars and late helium shell flash - born-again AGB - objects, discussed in Sect. 3.2) heat up while evolving at late phases. Models, as well as observations, show that $T_{\text {eff }}$ of novae can then reach values well above $10^{5} \mathrm{~K}$, giving rise to a nebular stage and a significant flux in X-rays. When the nuclear burning stops, the object decreases in luminosity as a very hot star. In the H-R diagram, the three objects, i.e. RV M31, V4332 Sgr and V838 Mon, evolved in the opposite direction to a nova. As discussed in Sect. 2, they evolved to lower $T_{\text {eff }}$ and faded as very cool stars. So far the largest luminosity decrease has been recorded for V4332 Sgr, which faded by a factor of $\sim 1500$ in 9 years, still being an M-type object. In our opinion, this type of evolution in luminosity and effective temperature is evidence that the V838 Mon type eruptions are not nove-like events. Below we discuss this and other points concerning the nova mechanism in more detail and in relation to individual objects.

Iben \& Tutukov (1992) have proposed a nova-like scenario to account for the M $31 \mathrm{RV}$ eruption. They consider a low-mass $\left(\sim 0.6 M_{\odot}\right)$ cold white dwarf in a short-period cataclysmic binary, which accretes at a very low rate $\left(\sim 10^{-11} M_{\odot} \mathrm{yr}^{-1}\right)$. This allows it to accumulate a relatively large mass $\left(\sim 0.005 M_{\odot}\right)$ before thermonuclear runaway. The authors argue that in this case the nova outburst would be energetic (maximum luminosity well above the Eddington limit) and the object can evolve to low effective temperatures due to a massive expanding 
envelope remaining optically thick for a few years. This scenario does not allow one to avoid the hot phase after the outburst. Sooner or later the expanding envelope becomes transparent and the steady burning white dwarf would have to be visible. The phase of steady burning is very long for low mass white dwarfs. From the results of Yaron et al. (2005) it can be inferred that for an $0.65 M_{\odot}$ white dwarf it lasts at least $\sim 100$ yr. The scenario also implies that the binary system is old $\left(\sim 10^{10} \mathrm{yr}\right)$ and that the main sequence companion is of low mass $\left(\$ 0.3 M_{\odot}\right)$, i.e. an M type dwarf.

In the case of M31 RV the scenario of Iben \& Tutukov cannot be conclusively confirmed nor definitively rejected. We know nothing about its progenitor. We have no data on the object for epochs later than 100 days after its discovery in eruption, so a hot phase, when the object is expected to be quite faint in optical, cannot, in principle, be excluded. However, the hot phase is likely to produce strong emission lines when the expanding matter becomes ionized. It seems rather unlikely that a nebular stage of M31 RV escaped detection in spite of numerous surveys for planetary nebulae done for M31 in the last decade.

In the case of V4332 Sgr the scenario of Iben \& Tutukov cannot be applied. The progenitor was most probably an $\mathrm{F}-\mathrm{K}$ type star. Thus, if identified as a donor secondary, it would imply a system in the upper range of the secondary mass $\left(\sim 1 M_{\odot}\right)$ of cataclysmic binaries. However, the $B-R$ colour from archive data discussed in Tylenda et al. (2005a) is uncertain so an $\mathrm{M}$ type progenitor, required by Iben \& Tutukov, cannot be definitively excluded. But then its absolute magnitude would be $M_{V} \gtrsim+9.0$, the distance to the object would be $\lesssim 300 \mathrm{pc}$ and the luminosity during outburst would be $\lesssim 150 L_{\odot}$. Such a low luminosity is obviously ruled out in any white dwarf thermonuclear event.

Thus, if continuing with the nova-type scenario in the case of V4332 Sgr, an $\sim 1 M_{\odot}$ secondary has to be accepted. This however implies a typical mass transfer rate $\left(\gg 10^{-11} M_{\odot} \mathrm{yr}^{-1}\right)$ and a typical nova outburst. Then there is no way to evolve to $M$ spectral types after the outburst maximum. Second, there is no way to avoid the nebular stage. The object is now $\sim 1500$ times fainter than at maximum so the white dwarf had to switch off nuclear burning and thus it had to have gone through a very hot phase in the past giving rise to a nebular stage. Although we have almost no data on the object between 1995-2002, it seems rather unlikely that its nebular stage would have escaped detection in spite of numerous surveys for emission-line objects (e.g. planetary nebulae) done in the last decade in the Galactic disc and bulge (galactic coordinates of V4332 Sgr are $\left.l=13^{\circ} .63, b=-9^{\circ} .40\right)$. A luminosity drop by a factor of 1000 on a time scale of a few years (note that the initial drop in lumonosity by a factor of 100 occured over 3 months, Martini et al. 1999) requires a massive white dwarf in the nova model, which in turn is expected to result in expansion velocities $\gtrsim 1000 \mathrm{~km} \mathrm{~s}^{-1}$ (e.g. Yaron et al. 2005). This is at least an order of magnitude higher than the value of $\sim 100 \mathrm{~km} \mathrm{~s}^{-1}$ observed in V4332 Sgr. Finally, the observed radius of the V4332 Sgr remnant in 2003, i.e. $\sim 5 R_{\odot}$ compared to $\sim 1 R_{\odot}$ for the progenitor (Tylenda et al. 2005a), implies that the whole (hypothetical) nova-like binary would still be embedded in a common envelope. Then how was the white dwarf able to extinguish nuclear burning if it still has an extended hydrogen-rich envelope?

The progenitor of V838 Mon was a rather hot and luminous star. If the object forms a (presumably very wide) binary with the B3 V companion identified by Munari et al. (2002a), then the progenitor was either a B2-3 main sequence star with a mass $\sim 8-10 \quad M_{\odot}$ or a pre-main-sequence star of $\sim 5 M_{\odot}$ (Tylenda et al. 2005b). In the former case, one can propose evolutionary paths in binaries in which a white dwarf can be formed from an initially more massive companion, especially if mass transfer took place between the binary components. However if the cooling time ( $\gtrsim 10^{7}$ years) necessary for the white dwarf to cease emitting significant ionizing flux (no significant ionization is observed in the diffuse matter surrounding V838 Mon), as well as to become able to produce a nova-type outburst, is taken into account, very little room remains for this possibility. The scenario seems to be very unlikely given the fact that we do not know of any cataclysmic variable having a B-type main sequence secondary. In the former case the situation is even worse. The age of a $\sim 5 M_{\odot}$ pre-main-sequence star can be estimated as $\sim 5 \times 10^{5}$ years. There is no way to produce and cool a white dwarf companion in such a short time.

If V838 Mon and the B3 V companion are observed at the same position in the sky due to a random coincidence then, apart from the above possibilities, the progenitor of V838 Mon could have been either a BA-type giant of mass $\gtrsim 2.5 M_{\odot}$ or a B-type post-AGB star (Tylenda et al. 2005b). In both cases a white dwarf companion cannot be excluded. In the former case the giant would be in a rapid expansion phase. Thus if mass transfer begins it would increase rapidly to rates close to, or even above, the Eddington limit for accretion on a white dwarf. The white dwarf would thus be bright and the whole system would remind a symbiotic star. In these conditions the hydrogen burning on the white dwarf surface would be ignited rather gently leading to an increase in the system brightness only by a factor of a few (compared to the giant luminosity plus the white dwarf accretion luminosity). V838 Mon increased in luminosity by a factor of $\sim 1000$.

The case of a B-type post-AGB star implies that a few thousand years ago the object was an AGB giant. Thus the only possibility to get a nova-type event is that the white dwarf companion was accreting from the AGB wind. However, in order to reach a nova peak luminosity above $10^{5} L_{\odot}$ the accretion rate would have to have been $\lesssim 10^{-10} M_{\odot} \mathrm{yr}^{-1}$ (Yaron et al. 2005). Taking a typical AGB mass loss rate of $10^{-6} M_{\odot} \mathrm{yr}^{-1}$ (note that at the end of $\mathrm{AGB}$, i.e. just before the post-AGB phase, mass loss is expected to be significantly higher than that) a wind velocity of $10 \mathrm{~km} \mathrm{~s}^{-1}$ and using Eq. (4.38) in Frank et al. (2002) one finds that the above accretion rate onto a $1 M_{\odot}$ white dwarf is possible if the binary separation is $\gtrsim 2 \times 10^{5} R_{\odot}$. Such a large binary separation is excluded. It would be $\gtrsim 100$ times larger than the maximum effective radius of V838 Mon during eruption (Tylenda 2005). Thus the post-AGB component would be visible all the time, including the decline phase. Yet the progenitor was not seen in the decline phase when V838 Mon became very cool and invisible in the optical (e.g. Munari et al. 2005). 
Thermonuclear outbursts can in principle supply a huge amount of energy (e.g., Sparks et al. 1978) with the maximum luminosity at peak of $\gtrsim 10^{5} L_{\odot}$ achieved by fast novae. In V838 Mon the maximum luminosity of $\sim 10^{6} L_{\odot}$ lasted $\sim 70$ days. Novae with a decline of $2 \mathrm{mag}$ in $t_{2} \sim 100$ days, have for their maximum visual luminosity $L_{V}(\max )<10^{5} L_{\odot}$, and more typically $L_{V}(\max ) \simeq 2 \times 10^{4} L_{\odot}($ Cohen 1985$)$.

The outflow velocity observed in V838 Mon, typically $\sim 150-400 \mathrm{~km} \mathrm{~s}^{-1}$, can be matched with slow novae, but not with fast novae. Some models calculated by Yaron et al. (2005) reach a nova peak luminosity of $\sim 10^{6} L_{\odot}$. However, the ejection speeds in these cases are $>2000 \mathrm{~km} \mathrm{~s}^{-1}$, much faster than observed in V838 Mon.

All the nova models in the extensive grid of Yaron et al. (2005) eject $<10^{-3} M_{\odot}$. In the case of the most luminous ones, i.e. having a maximum luminosity $\sim 10^{6} L_{\odot}$, it is even $\lesssim 5 \times 10^{-5} M_{\odot}$. Estimates for V838 Mon give mass loss $>5 \times$ $10^{-3} M_{\odot}$, most likely $\sim 0.01-0.1 M_{\odot}$. Simliar mass loss also probably occured in M $31 \mathrm{RV}$.

V838 Mon is embedded in diffuse matter which resulted in the spectacular light echo following the outburst. The light echo phenomenon is not a common feature of novae. This is what is expected. Fast (previous) ejecta should sweep up any circumstellar or interstellar matter from the vicinity of the nova. The light echo has been observed only in the case of the fast nova GK Per (Nova Per 1901) (Ritchey 1901; Couderc 1939). The echoing dust in GK Per was either interstellar in origin (Hessman 1989) or due to an old planetary nebula (Bode et al. 2004). Tylenda (2004) and Tylenda et al. (2005b) argue that the echoing matter in V838 Mon is interstellar. This is compatible with the idea that V838 Mon is a young object rather than an evolved nova-like system.

The strong infrared excess observed now in V4332 Sgr strongly suggests that the object is surrounded by a large (inner radius of $\sim 30$ central star radii) and luminous ( $\sim 15$ times brighter than the central star) accretion disc (Tylenda et al. 2005a). It is unlikely that a structure like this could have formed arround a nova-type binary.

The abundances usually are important in identifying the nature of an object. Simulations show that novae with very large peak luminosity are expected to eject highly processed matter with $Z$ up to $\sim 0.5$ (Yaron et al. 2005). As discussed in Sect. 2, the observed abundances in V838 Mon are compatible with unprocessed matter. Although there are no data on the abundances of key elements for novae, such as $\mathrm{C}, \mathrm{N}, \mathrm{O}, \mathrm{Mg}$ and $\mathrm{Ne}$, no strong molecular $\mathrm{CN}$ lines were observed in V838 Mon; strong $\mathrm{CN}$ lines are expected from novae at early stages (Pontefract \& Rawlings 2004).

As noted in Orio et al. (2003), the lack of measurable X-ray flux in V838 Mon is evidence against a nova-like runaway in this object.

\subsection{Helium shell flashes}

While in novae thermonuclear runaway occurs on a relatively cool white dwarf, in He-shell flashes the runaway process takes place in a shell at the outskirts of the core of a nuclear active star. This takes place in AGB and post-AGB stars. Before the flash the energy generation is maintained by H-burning that adds more helium to the He-shell, until conditions for ignition are reached. One of the characteristics of this process is that because of expansion and cooling, the H-burning is extinguished as the He-shell ignites. As a result, the surface luminosity typically varies only by a factor of 2-3 during the thermal pulse (Wood \& Zarro 1981; Vassiliadis \& Wood 1993; Herwig 2000). Thus there is no way to obtain an increase of $\sim 10^{3}$ in luminosity, as observed in V838 Mon and V4332 Sgr, from a thermally pulsating AGB star. In the case of V838 Mon the AGB hypothesis has to be rejected also on observational evidence. As shown in Tylenda et al. (2005b) the progenitor of this object was significantly hotter than a KM-type giant or supergiant.

As discussed in Tylenda et al. (2005b) it is possible (although unlikely) that the V838 Mon progenitor was a B-type post-AGB star. An He-shell flash, usually called a late He-shell flash, can also occur at this evolutionary stage. However, for the same reason as discussed above (an active $\mathrm{H}$-shell) only a moderate (at most a factor of 10) increase in brightness is possible (Iben 1984; Blöcker \& Schönberner 1997).

The only way to get a significant luminosity increase during a He-shell flash is the so-called very late He-shell flash or born-again AGB event which occurs on the cooling part of the post-AGB evolutionary track when the $\mathrm{H}$-shell is already extinguished (Iben 1984; Herwig 2001; Lawlor \& McDonald 2003). However in this case the object is expected to be very hot $\left(\sim 10^{5} \mathrm{~K}\right)$ before the flash and surrounded by a glowing planetary nebula. Indeed, objects identified as undergoing a (very) late He-shell flash, i.e. FG Sge, V4334 Sgr, V605 Aql, are surrounded by planetary nebulae. Nothing of this kind has been reported for the V838 Mon type objects. In the case of V838 Mon itself we know from its light echo that the object is surrounded by diffuse matter but this matter is not ionized (Orio et al. 2002; Munari et al. 2002b). For the same reason (lack of ionization of the light echo matter) the idea of Munari et al. (2005), namely that the eruption of V838 Mon was due to a thermonuclear shell flash in an evolved massive star (initial mass $\sim 65 M_{\odot}$ ) close to the Wolf-Rayet phase $\left(T_{\text {eff }} \simeq 5 \times 10^{4} \mathrm{~K}\right)$, has to be rejected (Tylenda et al. 2005b).

Another important point concerning the late He-shell flash is that after the initial excursion to high luminosities and low effective temperatures, the object is expected to repeat its previous evolutionary path toward high effective temperatures. The evolution is thus qualitatively the same as that of a nova at late stages. As discussed in Sect. 3.1 this type of evolution is in a sharp disagreement with the observed evolution of the V838 Mon type objects. This is one of the crucial arguments against interpreting these objects as undergoing a (very) late He-shell flash.

Finally, as noted in Kipper et al. (2004), the abundances observed in V838 Mon do not support the idea of a late Heshell flash. In particular all the objects identified as flashing post-AGB stars (FG Sge, V4334 Sgr, V605 Aql, as well as central stars of A 30 and A 78) are C-rich while all the V838 Mon type objects have $\mathrm{C} / \mathrm{O}<1$. 


\subsubsection{The born-again model of Lawlor}

Lawlor (2005) proposed a born-again AGB model including an episode of accretion to account for the V838 Mon eruption. The accretion is supposed to be due to a burst of mass loss from a main-sequence binary companion irradiated by the He-shell flashing white dwarf. The addition of accretion produces a second outburst, which the author identifies with the main eruption of V838 Mon that started in the first days of February 2002.

The arguments raised above against the very late He-shell flash also hold in the case of Lawlor's model. This model assumes a very hot progenitor and must evolve to high effective temperatures before final fading. Other important problems appear when comparing assumptions and results of the model with the observations of V838 Mon.

As the main observational test Lawlor (2005) calculated a visual light curve from his model and obtained a result qualitatively similar to the observed one assuming a distance of $6.3 \mathrm{kpc}$. However when calculating the model light curve Lawlor did not take into account interstellar extinction which in the case of V838 Mon is as large as $A_{V} \simeq 2.7$ (Munari et al. 2005; Tylenda 2005). Thus an agreement between the model and observed light curves can be obtained but for a distance as small as $1.8 \mathrm{kpc}$, which can be compared to e.g. a lower limit of 5-6 kpc obtained from the light echo expansion (Bond et al. 2003; Tylenda 2004; Tylenda et al. 2005b). For more reasonable distance estimates of $8-10 \mathrm{kpc}$ the model of Lawlor is systematically too faint by a factor of 20-30.

Another important problem is that the surface parameters of Lawlor's model just before the accretion event are $T_{\text {eff }} \simeq$ $40000 \mathrm{~K}$ and $R \simeq 1.5 R_{\odot}$. This phase is supposed to correspond to the pre-eruption phase of V838 Mon in January 2002. However, the observed parameters of V838 Mon during this phase were $T_{\text {eff }} \simeq 5000 \mathrm{~K}$ and $R \simeq 350 R_{\odot}$ (Tylenda 2005). This discrepancy ensures, as we discuss below, that Lawlor's idea of an irradiation-induced accretion event cannot occur in the case of V838 Mon.

Lawlor (2005) proposes that the irradiated companion is the B3 V companion discovered by Munari et al. (2002a). However this companion must be at a distance of at least $\sim 3000 R_{\odot}$ from V838 Mon (effective radius of V838 Mon at the epoch when the companion was discovered - Tylenda 2005) and most probably is much father away. Obviously, no important irradiation effects nor significant mass exchange can be expected at such a large distance between the components. Thus the only possibility is that the star identified as the progenitor of V838 Mon in Tylenda et al. (2005b) was irradiated by its hypothetical flashing white-dwarf binary companion. This star most probably was an early B-type main sequence star prior to the V838 Mon eruption. However, since the irradiation-induced mass transfer is supposed to occur during the pre-eruption of V838 Mon, i.e. in January 2002, the B-type star would have to be at a distance of at least $\sim 1600 R_{\odot}$ from V838 Mon (supposed to be the flashing white dwarf). Otherwise V838 Mon, inflated to $\sim 350 R_{\odot}$ in the pre-eruption phase, would fill up its Roche lobe and mass transfer would occur in the direction opposite to that required in the model of Lawlor. Obviously the B-type main sequence star would be deeply inside its Roche lobe, so in order to obtain significant mass loss from it (Lawlor's model requires accretion at a rate of $\sim 10^{-3} M_{\odot} \mathrm{yr}^{-1}$ ) the irradiated flux would have to be significantly greater than the surface flux of the B-type star. This would require an irradiation source as luminous as $\gtrsim 2 \times 10^{8} L_{\odot}$. In January 2002 V838 Mon had an effective temperature of $\sim 5000 \mathrm{~K}$ (Tylenda 2005 ; see also e.g. Kimeswenger et al. 2002; Rushton et al. 2005b), i.e. it was much cooler than an early B-type star. For obvious reasons a cooler object cannot significantly heat up a hotter one even if the cooler one is much more luminous.

As discussed in Sect. 2 V838 Mon lost $0.01-0.1 M_{\odot}$ of $\mathrm{H}$-rich matter during its eruption. There seems to be no way to get such a large mass loss from the model of Lawlor. The mass of the H-rich envelope of a white dwarf before a very late He-shell is $<10^{-3} M_{\odot}$ (Iben 1984) and $<10^{-4} M_{\odot}$ has been accreted in Lawlor's model. Intense mass loss from Lawlor's model would quickly expose highly processed matter at the surface. Neither in the 2002 eruption nor during the decline was there any observational indication of this. In particular, the $\mathrm{C} / \mathrm{O}$ ratio remained $<1$ all the time.

We can conclude that the model presented in Lawlor (2005) cannot be accepted as an explanation of the V838 Mon eruption. It is discrepant with the observations in too many points.

\section{The stellar merger model}

In the previous section we showed that neither the nova-like runaway nor the He-shell flash can account for the observed properties of the V838 Mon type eruptions. The only other way to liberate a significant amount of energy in stellar astrophysics is release of gravitational energy, i.e. gravitational contraction or accretion. Gravitational contraction powers protostars. It is possible, as discussed in Tylenda et al. (2005b), that the progenitor of V838 Mon was a pre-main-sequence star. However there is no known way in which an energy of $\gtrsim 3 \times 10^{46} \mathrm{erg} \mathrm{s}^{-1}$ could be released on a time scale of a month in a pre-main-sequence star.

Thus we have to consider accretion events. The more compact the accreting object, the more effective is the accretion in terms of energy liberated per unit mass accreted. Accretion onto a neutron star or a black hole, being the most effective, is however excluded as it would result in an X-ray source. Accretion onto a white dwarf would have to procede at a super-Eddington rate to approach the observed luminosities and would lead to a nova-type thermonuclear event with all the problems involved, as discussed in Sect. 3.1.

We are left with accretion on a main sequence star. In this case, to obtain a luminosity of $\sim 10^{6} L_{\odot}$ the accretion rate must be $\gtrsim 0.01 M_{\odot} \mathrm{yr}^{-1}$. Taking into account the total energy involved in the V838 Mon event, as estimated in Sect. 4.1, the main sequence star would have to accrete $\gtrsim 0.1 M_{\odot}$ on a time scale of months. An event like this is excluded in secular binary system evolution, as there is no mechanism that could result in the transfer of such a large amount of mass in such a short time. Young main sequence or pre-main-sequence stars are often surrounded by massive protostellar discs. Thermal instabilities in the disc, similar to those in dwarf novae, can transfer a significant mass from the disc to the central star. This is the 
mechanism believed to produce FU Ori type eruptions (Hartmann \& Kenyon 1996). The discs in these objects can transfer as much as $0.01 M_{\odot}$. However this occurs on a time scale of a hundred years.

Therefore it seems that the only way to get the accretion event required to explain the V838 Mon type eruptions is accretion of a low mass object onto a main sequence star. This is the reason why we proposed a stellar merger model in Soker \& Tylenda (2003) to explain the eruption of V838 Mon. At that time the distance to the object was believed to be $\sim 1 \mathrm{kpc}$. Therefore, the merging of two low-mass main-sequence stars was considered.

In the present paper the qualitative general scenario for the eruption of V838 Mon remains similar to that in Soker \& Tylenda (2003). We however change some details and update numerical values to be consistent with the new distance estimate and more recent data on the evolution of the object, in particular with the indication that the system is more massive and young. A general outline of the scenerio presented below (Sect. 4.1) is followed by simple simulations of the merger remnant in Sect. 4.2. A discussion of the observations within the merger scenario is given in Sect. 4.3.

\subsection{General considerations and estimates}

We assume, following Tylenda et al. (2005b), that the progenitor of V838 Mon was a main sequence star of $M_{1} \simeq 8 M_{\odot}$ or a somewhat less massive star in the pre-main-sequence phase. In the following we will call it the primary. Most likely it forms a wide binary with a similar star, seen as a B3 V companion in the decline phase of V838 Mon. It is possible that the system also had another component, or other components, of much lower mass. Interactions within the multiple system might have destabilizated the orbit of one of the low mass components causing it to enter a highly eccentric orbit and, finally, to interact with the primary. As the whole system is rather young, as discussed in Tylenda et al. (2005b), another possibility is that the V838 Mon progenitor and its presumably low mass companion are surrounded by a protostellar disc. Interactions between the disc and the low mass star might have caused migration of the latter and growth of the orbit eccentricity, as discussed, for instance, in Artymowicz et al. (1991) for stellar binaries or in Armitage \& Bonnell (2002) for migrating brown dwarfs arround low mass stars, leading to a merger with the primary. In the case of a primary at the end of the main sequence phase, the onset of fast expansion of the star might have contributed to triggering the merger process.

The mass of the accreted companion, $M_{2}$, in V838 Mon can be estimated from the total energy budget of the event. As discussed in Sect. 2, during the main outburst (January-midApril 2002) V838 Mon lost $\sim 2.5 \times 10^{46}$ erg in radiation and presumably $0.01-0.1 M_{\odot}$ in mass loss. The energy necessary to lift this matter from the surface of a star having $M_{1}=8 M_{\odot}$ and $R_{1}=5 R_{\odot}$ and to accelerate it to $300 \mathrm{~km} \mathrm{~s}^{-1}$ at large distances is $(0.4-4.0) \times 10^{47} \mathrm{erg}$. Tylenda (2005) estimated that the mass of the inflated envelope contracting in the decline phase was $M_{\mathrm{env}} \simeq 0.2 M_{\odot}$. This value was obtained assuming a $n=3 / 2$ polytropic model of the envelope. If a $n=3$ polytropic model is assumed, as discussed below, then the result would be $M_{\text {env }} \simeq 0.1 M_{\odot}$. The energy stored in such an envelope, i.e. a difference between the values obtained from Eq. (A.11) in Tylenda (2005) putting $R_{\text {env }}=2000 R_{\odot}$ and $R_{\text {env }}=R_{1}$, is $(2.5-6.0) \times 10^{47} \mathrm{erg}$. Thus the total energy involved in the V838 Mon event is $(3-10) \times 10^{47} \mathrm{erg}$. Equating this value to $\left(G M_{1} M_{2}\right) /\left(2 R_{1}\right)$ one obtaines $M_{2}=$ $0.10-0.33 M_{\odot}$.

The above analysis can be repeated assuming an A-type pre-main-sequence star progenitor discussed in Tylenda et al. (2005b), i.e. taking $M_{1}=5 M_{\odot}$ and $R_{1}=7.5 R_{\odot}$. In this case $M_{\text {env }}$ becomes $\sim 0.3 M_{\odot}$ and $\sim 0.15 M_{\odot}$ for the $n=1.5$ and $n=3$ polytropes, respectively. The mass of the accreted component is then obtained as $M_{2}=0.15-0.5 M_{\odot}$.

As discussed in Tylenda et al. (2005a) the case of V4332 Sgr can be accounted for by a collision of a planet-like component with a $\sim 1 M_{\odot}$ main sequence star.

Let us consider a grazing collision, rather than a headon collision, as more plausible in the case of a binary merger in an initial eccentric orbit. The gravitational plus kinetic energy of the binary system before merger is

$E_{\mathrm{orb}}=-\frac{G M_{1} M_{2}}{2 r_{\mathrm{p}}}(1-e)$,

where $r_{\mathrm{p}}$ is the orbital separation at periastron and $e$ is the eccentricity. For a grazing merger we have $R_{1} \lesssim r_{\mathrm{p}} \lesssim R_{1}+R_{2}$ where $R_{2}$ is the radius of the secondary. In the following we will use $r_{\mathrm{p}} \simeq R_{1}$.

We mostly consider low-mass main-sequence or pre-mainsequence stars as secondaries. Their structure can be well approximated by a polytropic star with an index $n=3 / 2$. Thus the total internal (gravitational plus thermal) energy of the secondary can be written as (see Eq. (A.2))

$E_{2}=-\frac{3}{7} \frac{G M_{2}^{2}}{R_{2}}$

It is reasonable to assume that in the case of a grazing collision the low mass secondary, if it is not significantly denser than the more massive primary, is disrupted in the merger process and forms an inflated envelope of the merger remnant. The primary, remaining almost undisturbed, forms a core of the remnant. This scenario is confirmed by the simulations presented in Sect. 4.2. The structure of the remnant envelope is determined by the distribution of the energy dissipated in the merger process. The latter is a complex process as demonstrated in numerical simulations (e.g. Lombardi et al. 1996, 2002). However, to a first approximation we can assume that the rate of the energy dissipation is proportional to the density. In this case the structure of the remnant envelope can be approximated by a uniform energy source model, which is close to an $n=3$ polytrope (see e.g. Cox \& Guili 1984). Indeed, the density distribution in the remnant envelopes obtained in our simulations discribed in Sect. 4.2 (not only in the cases shown in Fig. 1) can be well reproduced by the $n=3$ polytropic distributions (see Eq. (A4) in Tylenda 2005). For an outer radius of the envelope, $R_{\mathrm{env}}$, much larger than the inner one (assumed to be the radius of 


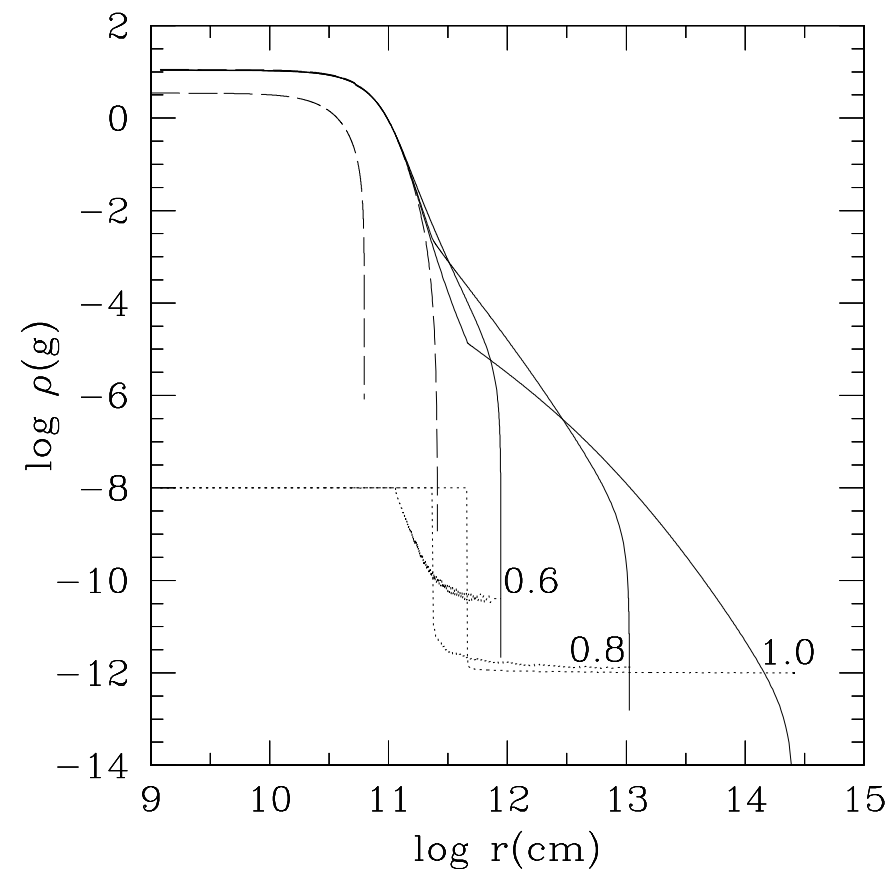

Fig. 1. Results of a grazing collision of two stars using the MMAS package. The initial system consists of an $8.0 M_{\odot}$ main sequence primary and an $0.3 M_{\odot}$ pre-main sequence secondary at an evolutionary age of $5.7 \times 10^{6}$ years. Dashed curves: density profiles of the stars (upper: primary - lower: secondary) before collision. Full curves: density profile in the merger product. Dotted curves: values of a parameter showing the origin of the matter in the merger product (-8: matter from the primary, -12 : matter from the secondary - see text). The curves (full and dotted) are marked with the value of $x_{\mathrm{p}}=r_{\mathrm{p}} /\left(R_{1}+R_{2}\right)$.

the primary, $R_{1}$ ) the total energy of the envelope, $E_{\text {env }}$, can be approximated by (see Eq. (A.17) in Tylenda 2005)

$E_{\text {env }} \simeq-\frac{G M_{1} M_{2}}{2 R_{1}} \frac{1}{\ln \left(R_{\text {env }} / R_{1}\right)}$.

The energy balance can be written as

$E_{\text {orb }}+E_{2}=E_{\text {env }}$,

which, using Eqs. (1)-(3), can be transformed to

$\ln \frac{R_{\mathrm{env}}}{R_{1}} \simeq\left[(1-e)+\frac{6}{7} \frac{M_{2}}{M_{1}} \frac{R_{1}}{R_{2}}\right]^{-1}$.

In the above energy balance we do not consider energy losses in form of radiation and mass loss. In the global energetics of the event they can be important, as shown in our estimates above for V838 Mon. However, these losses mostly take place in later phases of the evolution of the merger remnant, which occur on a thermal time scale. The merger is expected to occur on a dynamical time scale so for the initial structure of the merger remnant the losses are not expected to be important. This is consistent with numerical simulations of stellar mergers (e.g. Lombardi et al. 2002; see also Sect. 4.2).

Analyses of observations give $R_{\text {env }} / R_{1} \gg 1$. Maximum values of the photospheric radius in the case of V838 Mon reached $\sim 3000 R_{\odot}$ (Tylenda 2005). This however refers to freely expanding matter rather than to a hydrostatic remnant.
The latter was smaller but probably still as large as $\sim 2000 R_{\odot}$, as inferred from the photospheric radius observed during the early decline. For $R_{1}=5 R_{\odot}$ this corresponds to $R_{\text {env }} / R_{1} \simeq 400$. In the case of V4332 Sgr the envelope was probably less inflated but still as large as $R_{\text {env }} / R_{1} \simeq 100-150$ (Tylenda et al. 2005a).

Equation (5) shows that from a merger of two similar stars, e.g. two main sequence stars of not vary different mass, we do not expect a significantly inflated remnant. For instance, taking $M_{1}=M_{2}$ and $R_{1}=R_{2}$, Eq. (5) predicts $1.7 \lesssim R_{\text {env }} / R_{1} \lesssim 3.2$ for $0 \leq e \leq 1$. Note, however, that the cases of two similar stars are not particularly applicable to the above approach, which assumes an undisturbed primary and a largely inflated remnant envelope. Simulations, described in Sect. 4.2 for two $0.6 M_{\odot}$ ZAMS stars give $6.5<R_{\text {env }} / R_{1}<8.4$ for $0.5<r_{\mathrm{p}} /\left(R_{1}+R_{2}\right)<$ 1.0 , thus the remnants are more extended than predicted from Eq. (5) but still much less than observed.

Taking $M_{1}=8 M_{\odot}, M_{2}=0.3(0.1) M_{\odot}$, i.e. masses expected for V838 Mon, and the corresponding main sequence radii, i.e. $R_{1}=5 R_{\odot}$ and $R_{2}=0.35(0.15) R_{\odot}$, one still gets little expansion from Eq. (5), i.e. $R_{\text {env }} / R_{1} \lesssim 9.0(16.5)$. However the above set of stellar parameters is not realistic. From evolutionary considerations it can be concluded that at an age of an $8 M_{\odot}$ star leaving the main sequence, stars of masses $\lesssim 1.0 M_{\odot}$ still have to be in the pre-main-sequence phase. As can be seen from Eq. (5), larger values of $R_{2}$ would result in more inflated merger remnants. An $8 M_{\odot}$ star reachs the ZAMS at an age of $3.1 \times 10^{5}$ years (see Sect. 4.2). At this age a $0.3(0.1) M_{\odot}$ premain-sequence star is expected to have a radius of $\sim 2.5(1.3) R_{\odot}$, as estimated from Eq. (A.4). To get envelope extensions as large as $R_{\text {env }} / R_{1} \simeq 400$ Eq. (5) requires $R_{2} \gtrsim 0.95(0.32) R_{\odot}$.

If the V838 Mon progenitor was an early A-type pre-mainsequence star, we can take $M_{1} \simeq 5 M_{\odot}$ and $R_{1} \simeq 7.5 R_{\odot}$. The age of the star would be $\sim 5 \times 10^{5}$ years (see Sect. 4.2). At this age the radius of a $0.5(0.15) M_{\odot}$ pre-main-sequence star would be $\sim 3.5(1.5) R_{\odot}$. For these parameters (and assuming $e=1$ ) Eq. (5) predicts $R_{\text {env }} / R_{1} \simeq 230(2400$ ).

In the case of V4332 Sgr we can assume $M_{1}=1 M_{\odot}, R_{1}=$ $1 R_{\odot}$ and $R_{2}=0.1 R_{\odot}$ (radius typical for brown dwarfs and Jupiter-like planets). Then inflations of $R_{\text {env }} / R_{1} \simeq 150$ can be obtained from Eq. (5) if $M_{2} \lesssim 0.02 M_{\odot}$.

Even in the most favorable cases, i.e. when the term $\left(M_{2} / M_{1}\right)\left(R_{1} / R_{2}\right)$ in Eq. (5) is negligible, $e \gtrsim 0.8$ is required to get the remnant extension as large as $R_{\text {env }} / R_{1} \gtrsim 150$.

\subsection{Numerical simulations}

We have carried out simulations of a merger of two stars with parameters expected for V838 Mon. We used the MMAS (version 1.6) package described in Lombari et al. (2002, 2003) and available at http: /faculty.vassar .edu/ lombardi/mmas/. This code, based on simple physical arguments and algorithms, allows one to obtained a one dimensional structure of the collision product without hydrodynamic simulations. It still accounts for shock heating, mass loss and fluid mixing. The remnants returned by MMAS are in hydrostatic equilibrium but before thermal relaxation. The code 
explicitely assumes $e=1.0$ before collision so its results can be considered as reasonable for highly eccentric binary orbits.

Following the discussion in Sect. 4.1 we have considered an $8.0 M_{\odot}$ main sequence primary which accretes an $0.3 M_{\odot}$ pre-main-sequence secondary. As argued in Sect. 4.1, a grazing merger is more likely than a headon collision, so we discuss cases with $0.5<x_{\mathrm{p}} \leq 1.0$, where $x_{\mathrm{p}}=r_{\mathrm{p}} /\left(R_{1}+R_{2}\right)$.

The structure of the primary before merger was obtained from the TYCHO (version 6.0) stellar evolution code (Young et al. 2001) available at http : //chandra . as . arizona . edu/ $\sim$ dave/tycho-intro.html. Starting from an initial (premain-sequence) model the $8.0 M_{\odot}$ star reaches the ZAMS at an age of $3.15 \times 10^{5}$ years. For our simulations we have taken the stellar structure at $5.7 \times 10^{6}$ years. The star is well on the main sequence with $\sim 10 \%$ of hydrogen burnt in the core and an outer radius $R_{1}=3.7 R_{\odot}$.

The structure of the secondary has been approximated by a $n=3 / 2$ polytrope, as discussed in Appendix A. Using Eq. (A.4) one gets that at an age of $5.7 \times 10^{6}$ years the radius of the $0.3 M_{\odot}$ secondary is $R_{2} \simeq 0.9 R_{\odot}$.

Density profiles for the stars (before merger) modelled as explained above are shown with dashed curves in Fig. 1. Full curves present the density profile of the merger products obtained with MMAS. Three cases of $x_{\mathrm{p}}=0.6,0.8$ and 1.0 are shown. We have defined a parameter to which values of -8 and -12 have been assigned for the matter in the primary and secondary, respectively, before merger. MMAS sorts and mixes the matter from both components after the merger event so the returned value of this parameter allows us to see from which component and in what proportion the matter comes at a given radius of the remnant. This result is shown with dotted curves in Fig. 1.

In the case of a main sequence star the density gradient is relatively flat in the interior, that quickly drops when approaching the star surface. Therefore if the star suffers a grazing collision with a low mass pre-main sequence star its interior structure remains almost intact (see Fig. 1). Only outer regions are seriously disturbed and together with the matter from the destroyed secondary they form an inflated envelope ${ }^{1}$. The outer radius of the envelope is very sensitive to $x_{\mathrm{p}}$ and it becomes as large as $3.7 \times 10^{3} R_{\odot}$ in the case of $x_{\mathrm{p}}=1.0$ shown in Fig. 1 . Note that Eq. (5) predicts $R_{\text {env }} \simeq 7 \times 10^{3} R_{\odot}$ which is in a fair agreement with the simulations. The mass of the inflated envelope is $\sim 0.30 M_{\odot}$. For $x_{\mathrm{p}} \gtrsim 0.8$ the remnant envelope is predominantly formed from the disrupted secondary. The simulations predict $\sim 0.015 M_{\odot}$ lost from the system, for $x_{\mathrm{p}} \gtrsim 0.8$ almost entirely from the secondary.

We have also carried out simulations for the same stars as in Fig. 1 but at an age of $3 \times 10^{7}$ years as well as for two pre-mainsequence stars of 5.0 and $0.3 M_{\odot}$ at an age of $5 \times 10^{5}$ years. In the former case the $8 M_{\odot}$ primary just started to move from the main sequence and the radii of the components were $R_{1}=9 R_{\odot}$ and $R_{2}=0.6 R_{\odot}$. In the latter case the effective temperature of the $5 M_{\odot}$ primary was $\sim 10000 \mathrm{~K}$, i.e. corresponding to

\footnotetext{
1 The situation is qualitatively different for collisions approaching headon, i.e. $x_{\mathrm{p}} \lessgtr 0.4$. Then most of the secondary dives into the primary centre and forms the core of the collision product.
}

an $\sim$ A0 spectral type discussed in Tylenda et al. (2005b). The stellar radii were $R_{1}=8 R_{\odot}$ and $R_{2}=2 R_{\odot}$. The results were very similar to those displayed in Fig. 1 . In particular the radii of the obtained remnants were $10^{2}-10^{4} R_{\odot}$.

In all the cases discussed the density distribution in the inflated envelope of the remnant can be well approximated by a polytropic envelope (see Appendix in Tylenda 2005) with an index $n=3$.

\subsection{Discussion}

As we have shown using simple estimates in Sect. 4.1 and simulations in Sect. 4.2, it is possible to obtain merger remnants with radii similar to those observed in V838 Mon and V4332 Sgr. These results should be verified with more sophysticated modelling. As noted in Sect. 4.2 the MMAS code is a simple algorithm. It has been calibrated on SPH simulations of mergers of two low-mass $\left(0.4-0.8 M_{\odot}\right)$ main-sequence stars. For the star parameters used in Sect. 4.2 the code works at its limits and the results are probably subject to considerable uncertainties.

Some important processes are not taken into account in the MMAS code. These include effects of radiation transfer and angular momentum. Radiation pressure becomes particularly important if the luminosity is super-Eddington. This was certainly the case in V838 Mon. In February-March 2002 the observed luminosity was above the Eddington value (Tylenda 2005), which means that the rate of energy dissipation deep in the envelope must have been super-Eddington by a larger factor. A part of energy went to expansion of the envelope and mass loss. Also energy diffusion processes in the envelope work in the sense that the observed eruption lasts longer, while the maximum luminosity is lower, compared to the original energy burst. Indeed, an analysis done in Tylenda (2005) suggests that the main eruption observed in February-March 2002 was generated on a time scale of a few days at the end of January 2002.

The rate of energy dissipation in a merger event can be estimated from

$L_{\mathrm{diss}} \simeq \frac{1}{2} \rho v^{3} \pi R_{2}^{2}$

where $v$ is the velocity of the accreted component of radius $R_{2}$ moving in an ambient medium of density $\rho$. Assuming that the merger takes place in the outer layers of the primary and that $v$ is close to the Keplerian velocity, i.e. $v \simeq \sqrt{G M_{1} / R_{1}}$, Eq. (6) becomes

$$
\begin{aligned}
L_{\mathrm{diss}} \simeq & 3 \times 10^{10} L_{\odot}\left(\frac{\rho}{0.1 \mathrm{~g} \mathrm{~cm}^{-3}}\right)\left(\frac{M_{1}}{8 M_{\odot}}\right)^{3 / 2} \\
& \left(\frac{R_{1}}{5 R_{\odot}}\right)^{-3 / 2}\left(\frac{R_{2}}{1 R_{\odot}}\right)^{2} .
\end{aligned}
$$

This is a very rough estimate but shows that in the case of mergers simulated in Sect. 4.2 the energy is expected to be dissipated at a very high rate, orders of magnitude above the Eddington limit. This can have important effects on the structure of the remnant and mass loss. In particular the total mass lost in the 
merger can be significantly higher than the values predicted from dynamical effects only in the MMAS code $\left(0.01-0.02 M_{\odot}\right.$ in our cases).

The evolution of a merger remnant can be quite easily predicted, at least qualitatively. The high energy sources will quickly decline after the merger. The rate of the energy transported from the base of the inflated envelope will soon become limited to the luminosity of the central star, which is much less than the luminosity radiated away from the photosphere. As a result, the matter in the envelope, which did not achieve escape velocity during the merger eruption, has to contract under the gravity of the central star. In this way the internal energy of the contracting envelope can be liberated at a rate necessary to balance the photospheric energy loss.

A transition between the eruption phase (dominated by expansion of the matter) and the decline phase (dominated by gravitational contraction of the envelope) is expected to occur on a dynamical time scale, which is

$t_{\mathrm{d}} \simeq\left(\frac{R_{\mathrm{env}}^{3}}{2 G M_{1}}\right)^{1 / 2}$.

For the observed radius of V838 Mon at the beginning of the decline, $R_{\mathrm{env}} \simeq 2000 R_{\odot}$, (and assuming $M_{1}=8 M_{\odot}$ ) Eq. (8) gives $t_{\mathrm{d}} \simeq 1$ year. Taking $R_{\mathrm{env}} \simeq 150 R_{\odot}$ and $M_{1}=1 M_{\odot}$ as the parameters for V4332 Sgr (Tylenda et al. 2005a) one gets $t_{\mathrm{d}} \simeq 1$ month. During this transition phase the photospheric regions are deprived of energy supply (merger energy is declining, contraction not yet effectively started) so they must cool down due to radiative processes. In V838 Mon this phase was observed over $\sim 200$ days since $\sim 10$ April 2002. As can be seen from Fig. 2 in Tylenda (2005), the object kept the effective radius between $2000-3000 R_{\odot}$, its effective temperature dropped from $\sim 3600 \mathrm{~K}$ to a minimum value of $\sim 1800 \mathrm{~K}$, while the luminosity declined by more than an order of magnitude. In the case of V4332 Sgr the transition phase probably started on 5 April 1994 (Tylenda et al. 2005a). During the subsequent three months the object dropped by a factor of $\sim 100$ in luminosity, reaching an effective temperature as low as $\sim 2300 \mathrm{~K}$ (Martini et al. 1999).

In later phases the evolution of a merger remnant will be governed by the gravitational contraction of its inflated envelope. Thus the main processes are here the same as in protostars. As a result we expect that on the HR diagram the object should decline along a Hayashi track. However the time scale of evolution of the merger remnant must be much shorter than that of a protostar, as instead of the whole star, only a small mass envelope is involved in the contraction. As shown in Tylenda (2005) and Tylenda et al. (2005a) the observed evolution of V838 Mon for 2.5 years after its 2002 eruption and the observed state of V4332 Sgr $\sim 9$ years after its 1994 eruption are consistent with this prediction.

This behaviour of V838 Mon type objects in initial decline resemble the expected evolution of supernova type Ia companions as discussed in Podsiadlowski (2003). These stars, when puffed up to large radii and luminosities by the supernova explosion, due to a very short thermal time scale are expected to enter into the "forbidden" Hayashi region and then decline rapidly.
Angular momentum is not taken into account when determining the remnant structure in the MMAS code. The code however provides the expected angular momentum distribution in the remnant. In our cases the ratio of the resultant specific angular momentum to the local Keplerian value is roughly constant throughout the inflated envelope and is typically $0.10-0.15$. This may have important effects on the structure of the remnant, particulary on its evolution with time. The above result means differential rotation of the envelope with the rotation velocity increasing inward as $\sim r^{-1 / 2}$. Radial motions, for instance convection, would transport angular momentum outward, thus accelerating rotation in outer layers. This may lead to an expansion of the envelope and/or intensified winds from the remnant in the equatorial directions. In later epochs, when the envelope as a whole is expected to contract, the angular momentum may prevent equatorial regions from significant contraction. Thus some matter can be left at larger distances, forming a Keplerian ring or disc arround the contracting central object. As discussed in Tylenda et al. (2005a) this is a likely explanation of the origin of cold matter seen in the emission molecular bands and atomic lines in the spectrum of V4332 Sgr.

One of the principal observational characteristics of an eruptive object is its light curve. V838 Mon displayed a quite complex light curve in January-April 2002, which can be interpreted as a sequence of three consecutive outbursts. This led Retter \& Marom (2003) to propose that the V838 Mon eruption was due to a red giant that swallowed three planets. This idea however suffers from several important shortcomings. First, as shown in Tylenda et al. (2005b) the progenitor of V838 Mon was not a red giant. Second, the energy budget of the V838 Mon eruption in Sect. 4.1 is such that a mass two orders of magnitude larger than three Jupiter-like planets is required to account for it. Third, it seems impossible to propose a scenario in which three planets would fall onto the central star one by one on a time scale of months. A study in Tylenda (2005) shows that if a difference in propagation time between the second (observed in February 2002) and the third (observed in March 2002) outburst is taken into account then the conclusion is that the two outbursts were generated on a time span of a few days only. The whole outburst can be divided into two main phases. The first one, observed in January 2002 and called pre-eruption in Tylenda (2005), was marked by a relatively static photosphere of $\sim 350 R_{\odot}$. The second one was observed in February-April 2002, called eruption, and was marked by an expansion of the photosphere from $\sim 350$ to $\sim 3000 R_{\odot}$.

The question that arises is how this multi-outburst light curve of V838 Mon can be explained in our merger model. Two general interpretations can be proposed to account for it. The first one is that the event involved accretion of two companions on a time span of a month. The second is that it was a single companion that collided and merged with the V838 Mon progenitor but that the merger event proceeded in multiple phases.

Accretion of two companions can occur in two scenarios. One possibility is that orbits of two low-mass companions had been disturbed, say due to a destabilizing event in the V838 Mon system, in such a way that the two companions collided with the primary star one after the other. Another 
possibility is that only one companion had been disturbed in its orbit so it collided with the primary but the merger event destabilized another companion causing its merger with the primary a month after the first one.

A more detailed scenario in the former case is as follows. The first companion collided with the primary star at the end of December 2001 and initiated the pre-eruption phase. As a results an envelope inflated to $\sim 350 R_{\odot}$ was formed. If there were another companion orbiting the primary at a distance much smaller than the radius of the envelope then it would become engulfed by the envelope. Drag forces between the orbiting companion and the envelope caused the companion to spiral in, so after a month it reached dense regions of the primary, more or less undisturbed by the accretion of the first companion. Then the second companion was disrupted, liberating its orbital energy which resulted in the main eruption started at the begining of February 2002. However, as we show below, it is very difficult, perhaps even impossible, to get a spiraling time as short as 1 month for realistic parameters in this scenario.

The luminosity of V838 Mon in the main eruption was $\sim 20$ times higher than that in the pre-eruption. This implies that the second companion was more massive than the first one by a factor similar to the luminosity ratio. Thus we can assume that the mass of the first companion was $\sim 0.01 M_{\odot}$. Let us approximate the structure of the envelope resulting from its disruption by a polytropic model (see Appendix in Tylenda 2005 ). The radius of the envelope was $\sim 350 R_{\odot}$, as observed in the pre-eruption phase, while its mass can be assumed to be comparable to the mass of the distrupted companion. Taking the mass and radius of the primary, $M_{1}=8 M_{\odot}$ and $R_{1}=5 R_{\odot}$, we can estimate a density at the base of the envelope, $\rho_{0}$, from Eq. (A.5) in Tylenda (2005). In the case of the polytropic index $n=3 / 2$ one gets $\rho_{0} \simeq 3 \times 10^{-7} \mathrm{~g} \mathrm{~cm}^{-3}$, while for $n=3$ the result is $\rho_{0} \simeq 1.5 \times 10^{-5} \mathrm{~g} \mathrm{~cm}^{-3}$. Now we can use Eq. (B.7) to estimate the time necessary for the second companion to spiral in, $t_{\mathrm{s}}$. Assuming $M_{2} \simeq 0.3 M_{\odot}, R_{2} \simeq 0.9 R_{\odot}$ (as in Sect. 4.2) and taking a rather lower limit to the orbital separation of $\sim 2 R_{1}$, i.e. putting $r_{0} / R_{0} \simeq 2.0$ in Eq. (B.7), the result is $t_{\mathrm{s}} \simeq 150$ and 6 years for $n=3 / 2$ and 3 , respectively. As can be seen from Eq. (B.7) $t_{\mathrm{s}}$ strongly increases with increasing values of $r_{0} / R_{0}$, e.g. for $r_{0} / R_{0}=10$ the in-spiral time becomes 5000 and 2000 years, respectively.

Another point is that during the spiraling phase the companion releases energy $\sim\left[G M_{1} M_{2}\left(1-R_{0} / r_{0}\right)\right] /\left(2 R_{0}\right)$ which for $R_{0} / r_{0} \ll 1$ becomes comparable to the energy released during the disruption of the companion at $R_{0}$. Thus even if it were possible to spiral in the companion over a month, the energy released during the pre-eruption phase would be comparable to that in the eruption phase. In the case of V838 Mon the ratio of the energy radiated away during the pre-eruption phase (January 2002) to that in the eruption phase (February-midApril 2002) was $\sim 0.03$.

Therefore we can conclude that if the 2002 eruption of V838 Mon was due to accretion of two star-like objects the only possibility seems to be that the two bodies arrived from outside on very similar trajectories: the lower-mass one was followed by the more-massive one with a month between them. It could have been a low-mass binary (e.g. a $\sim 0.3 M_{\odot}$ star orbited by a $\sim 0.01 M_{\odot}$ brown dwarf) "injected" by dynamical effects from the periphery of the V838 Mon system or from outside it.

In our opinion, the second interpretation is more reasonable. The outburst was likely due to the merger of a single companion but that the merger proceeded in multiple phases. In several numerical simulations of star collisions described in the literature this is indeed the case (e.g. Lombardi et al. 1996; Freitag \& Benz 2005; see also animations available at http://www . manybody . org/modest). The components, often partly disrupted after the first encounter, leave each other on a highly eccentric orbit and collide again at the return. This may be repeated, sometimes several times, with a rapidly decreasing time between the encounters. Thus a plausible interpretion of the light curve of V838 Mon is as follows. The first major encounter betweeen the low-mass companion and the progeniotor of V838 Mon happened at the end of December 2001 and produced the pre-eruption phase. The companion probably became partly disrupted and only a small part of the available energy was dissipated. Matter significantly shocked during the first encounter formed the pre-eruption envelope. Most of the matter from the companion collided with the primary after a month (note that the dynamical time scale - Eq. (8) - for the observed size of the envelope in the pre-eruption is $\sim 30$ days) and once again after a few days. This produced the main eruption with the double-maximum structure in the light curve observed in February and March 2002 and two shells in mass loss found by Tylenda (2005).

Before the main encounters, which led to the observed outburst, it is possible that there had been a series of minor encounters due to the companion passing by the periastron. This could have led to minor outbursts and a long-term increase in brightness before the main outburst. In the case of V838 Mon we have no observational data between 1994 and the outburst discovered at the beginning of 2002. Before 1994 the object had remained constant, at least for $\sim 55$ years (Goranskij et al. 2004). In the case of V4332 Sgr, however, Kimeswenger (2005) shows that the object started to rise several years before the outburst observed in 1994. This can be interpreted as a result of minor interactions before the final merger. If V4332 Sgr is a Galactic buldge object, then its progenitor was a G-type giant, as discussed in Tylenda et al. (2005a). Then the merger process may have occured well below the giant photosphere. Before the merger, when the companion was spiraling in the giant envelope, the brightness of the object was expected to steadily increase, as can be seen from Eq. (B.9).

We now discuss the observed abundances in the V838 Mon type objects. As discussed in Sect. 2, there is no observational indication that the matter observed in the objects was significantly processed by nuclear burning. In our merger model the observed matter is primarily due to the disrupted low-mass secondary. In the case of V838 Mon we argue that it was a lowmass pre-main-sequence star so no significant nuclear processing took place inside it. In particular this concerns the observed abundance of Li. Low-mass stars of masses $\lesssim 0.5 M_{\odot}$ are not expected to have significantly burnt $\mathrm{Li}$ at an age of $\lesssim 10^{7}$ years (Chabrier \& Baraffe 1997; also our own modelling done with the TYCHO code). Even if some mass from the primary had been mixed into the remnant it should have no significant 
effect on the surface $\mathrm{Li}$ abundances. $\mathrm{Li}$ is expected to remain unburnt in outer layers of an $8 M_{\odot}$ main-sequence or a $5 M_{\odot}$ pre-main-sequence star.

\section{Summary and discussion}

Table 1 summarizes our discussion of the models compared with the observations. For clarity it is restricted to the observed properties of V838 Mon only. Column (1) lists the principal observational properties of the object. Comments on how these properties can be explained in the classical nova, born-again AGB (very late He-shell flash) and stellar merger models are given in Cols. (2)-(4), respectively. In the table we do not discuss the idea of an evolved very massive star proposed in Munari et al. (2005). This is mainly because this idea is ruled out by the observed lack of noticeable ionization of the matter seen in the light echo (Tylenda et al. 2005b). There is no available model based on this idea so it is not clear what observational properties are predicted from it.

Table 1 shows that presently the merger model is the most promising one for V838 Mon and the other objects of the same type. Both classical nova and He-shell flash models seem to be ruled out by too many contradictions with the observed properties of the objects.

The main drawback of our merger model is that it is based on estimates and approximate considerations. The simple simulations presented in Sect. 4.2 only demonstrate that an inflated envelope can be formed from a merger. Realistic simulations should provide a three-dimensional structure of the inflated envelope, its evolution after merger, and the expected light curve. This would however be a very complex task involving threedimensional radiation-hydrodynamics. We hope that this paper will stimulate research in this direction.

So far we have seen three events of the V838 Mon type: two in our Galaxy, one in M31. A binary population synthesis done in Han et al. (1995) shows that 1-2 stellar mergers are expected in our Galaxy per 10 years. This result considers only binaries with stellar components having masses $\geq 0.8 M_{\odot}$. Thus if multiple systems, lower mass stars, brown dwarfs and massive planets were also considered, two events in our Galaxy in a time span of 8 years are not surprising. What is suprising is that no event of this kind had been reported (in our Galaxy) before the discovery of V4332 Sgr. Kato (2003) suggests that Nova CK Vul 1670 might have been a stellar merger event. Bond \& Siegel (2005) mention that Nova V1148 Sgr 1943 had a late-type spectrum thus being a possible object of the V838 Mon type. It is quite probable that some V838 Mon type objects remain hidden among stars catalogued as classical nova or nova-like.

Mergers of massive stars are considered as a possible way to form very high mass stars. Bally \& Zinnecker (2005) have discussed expected observational appearances of mergers involving massive stars. These events can release up to $10^{51} \mathrm{erg}$ and reach a peak luminosity above $10^{7} L_{\odot}$. They argue that an event of this kind happened $\sim 500$ years ago in the Orion molecular cloud. Their study as well as our work shows that stellar mergers are important not only to understand the evolution of binaries or the origin of blue stragglers. Due to the large amount of energy released on a short time scale they can also produce spectacular events, easy to observe even beyond our Galaxy.

Acknowledgements. The research reported in this paper has partly been supported by the Polish State Committee for Scientific Research through a grant No. 2 P03D 002 25, as well as by the Israel Science Foundation. The authors thank to the referee ( $\mathrm{Ph}$. Podsiadlowski) for very useful and constructive comments.

\section{Appendix A: Low mass pre-main-sequence stars}

Low mass pre-main-sequence stars are convective. In this case, for given values of the star mass, $M_{*}$, and radius, $R_{*}$, the stellar structure can be obtained from a solution of the Lane-Emden equation with a polytropic index $n=3 / 2$ (for detailes see e.g. Cox \& Giuli 1984).

A pre-main-sequence star radiates mainly due to its gravitational contraction, so its lifetime, $t_{\mathrm{pms}}$, can be estimated from (see e.g. Stahler 1994)

$t_{\mathrm{pms}} \simeq \frac{-E_{*}}{L_{*}}$.

Here $E_{*}$ is the total (gravitational plus internal) energy of the star, which, for an $n=3 / 2$ polytrope, is (see e.g. Cox \& Giuli 1984)

$E_{*}=-\frac{3}{7} \frac{G M_{*}^{2}}{R_{*}}$

while the luminosity, $L_{*}$, is

$L_{*}=4 \pi R_{*}^{2} \sigma T_{*}^{4}$,

where $T_{*}$ is the stellar effective temperature. Combining the above equations one gets

$$
\begin{aligned}
R_{*}= & f_{\mathrm{pms}}\left(\frac{3 G M_{*}^{2}}{28 \pi \sigma T_{*}^{4} t_{\mathrm{pms}}}\right)^{1 / 3} \\
= & 2.56 f_{\mathrm{pms}}\left(\frac{M_{*}}{0.3 M_{\odot}}\right)^{2 / 3}\left(\frac{T_{*}}{3000 \mathrm{~K}}\right)^{-4 / 3} \\
& \left(\frac{t_{\mathrm{pms}}}{10^{6} \mathrm{yrs}}\right)^{-1 / 3} R_{\odot}
\end{aligned}
$$

where $f_{\text {pms }}$ accounts for an uncertainty due to Eq. (A.1). Comparing results of evolutionary model calculations for low mass pre-main-sequence stars (Stahler 1994, our own models calculated with TYCHO) with the predictions from Eq. (A.4) we have found $f_{\mathrm{pms}}=0.6-0.8$.

\section{Appendix B: Spiraling in a polytropic envelope}

Let us consider a companion of mass $M_{2}$ and radius $R_{2}$ that orbits inside a non-rotating envelope of a primary star of mass, $M_{1}$, at a distance, $r$, from the star centre. We assume that $M_{2} \ll$ $M_{1}$ and that the density in the envelope, $\rho$, is much lower than the mean density of the companion, $\rho_{2}=\left(3 M_{2}\right) /\left(4 \pi R_{2}^{3}\right)$. Thus we can neglect effects of accretion or evapration processes on 
Table 1. V838 Mon versus models.

\begin{tabular}{|c|c|c|c|}
\hline $\begin{array}{l}\text { Observed property } \\
\text { (1) }\end{array}$ & $\begin{array}{l}\text { Classical nova } \\
\text { (2) }\end{array}$ & $\begin{array}{l}\text { Born-again AGB } \\
\text { (3) }\end{array}$ & $\begin{array}{l}\text { Stellar merger } \\
\text { (4) }\end{array}$ \\
\hline $\begin{array}{l}\text { Increase by factor } \sim 10^{3} \\
\text { in luminosity }(\Delta V \simeq 7.5)\end{array}$ & $\begin{array}{l}(+)^{a} \text { Possible, although } \\
\text { usually larger }\end{array}$ & $\begin{array}{l}(+) \text { Possible if starts well } \\
\text { down the cooling track }\end{array}$ & $(+)$ Easy to obtain \\
\hline $\begin{array}{l}\text { Multi-outburst } \\
\text { light curve }\end{array}$ & $\begin{array}{l}(+) \text { Compatible with } \\
\text { a slow nova }\end{array}$ & $\begin{array}{l}\text { (-) Cannot be explained } \\
\text { (Lawlor's accretion episode } \\
\text { cannot work) }\end{array}$ & $\begin{array}{l}(+) \text { Multiple phase } \\
\text { merger process }\end{array}$ \\
\hline $\begin{array}{l}\text { Fading as a very } \\
\text { cool supergiant }\end{array}$ & $\begin{array}{l}\text { (-) Contrary to expected } \\
\text { (decline at } T_{\text {eff }} \gtrsim 10^{5} \mathrm{~K} \text { ) }\end{array}$ & $\begin{array}{l}\text { (-) Contrary to expected } \\
\left.\text { (decline at } T_{\text {eff }} \gtrsim 10^{5} \mathrm{~K}\right)\end{array}$ & $\begin{array}{l}\text { (+) Contraction along } \\
\text { the Hayashi track }\end{array}$ \\
\hline $\begin{array}{l}L \simeq 10^{6} L_{\odot} \\
\text { over } \sim 70 \text { days }\end{array}$ & $\begin{array}{l}(-) \text { Too luminous for a slow } \\
\text { nova, too long for a fast nova }\end{array}$ & $\begin{array}{l}(-) \text { Too luminous } \\
\text { and too fast }\end{array}$ & $\begin{array}{l}(+) \text { Merger of } \sim 0.3 M_{\odot} \\
\text { and } \sim 8 M_{\odot} \text { stars }\end{array}$ \\
\hline $\begin{array}{l}\text { \solar abundances } \\
([\mathrm{Fe} / \mathrm{H}] \simeq-0.3)\end{array}$ & $\begin{array}{l}(-) \sim 10 \times \text { solar abundances } \\
\text { expected }\end{array}$ & $\begin{array}{l}(-) \text { Enrichment by } \\
\text { dredge-up expected }\end{array}$ & $\begin{array}{l}(+) \text { Young stars at } \\
\text { galactic outskirts }\end{array}$ \\
\hline $\begin{array}{l}\text { Outflow velocity } \\
200-500 \mathrm{~km} \mathrm{~s}^{-1}\end{array}$ & $(+)$ Observed in slow novae & (-) Too fast & $\begin{array}{l}(+) \sim \text { escape velocity } \\
\text { from a MS star }\end{array}$ \\
\hline B-type progenitor & (-) Cannot work & $\begin{array}{l}(-) \text { Too cool for a star } \\
\text { before born-again AGB }\end{array}$ & (+) B-type MS primary \\
\hline $\begin{array}{l}\text { Association with } \\
\text { a young (B3 V) star }\end{array}$ & $\begin{array}{l}(-) \text { too little time to form } \\
\text { and cool a WD }\end{array}$ & $\begin{array}{l}\text { (-) Incompatible with } \\
\text { a post-AGB progenitor }\end{array}$ & $(+)$ Progenitor of similar type \\
\hline $\begin{array}{l}\text { Circumstellar } \\
\text { non-ionized matter }\end{array}$ & $\begin{array}{l}\text { (-) Past eruptions expected } \\
\text { to sweep up the enviroment }\end{array}$ & $\begin{array}{l}\text { (-) Past AGB wind } \\
\text { expected to be ionized }\end{array}$ & (+) Part of an ISM region \\
\hline
\end{tabular}

$a$ (+) can be explained by the model; (-) cannot (very difficult to) be explained by the model.

the mass of the companion. Then we can assume that the companion moves with a Keplerian velocity, $v=\sqrt{G M_{1} / r}$, so its energy (gravitational plus kinetic) is

$E=-\frac{G M_{1} M_{2}}{2 r}$

Due to drag forces the companion loses its energy at a rate which we approximate by

$\frac{\mathrm{d} E}{\mathrm{~d} t} \simeq-\pi R_{2}^{2} \rho v \frac{v^{2}}{2}$

Taking a time derivative of Eq. (B.1) and substituting it in Eq. (B.2) one gets the spiraling rate

$\frac{\mathrm{d} r}{\mathrm{~d} t}=-\frac{\pi R_{2}^{2}}{M_{2}} \rho\left(G M_{1} r\right)^{1 / 2}$.

We assume that the structure of the envelope is given by a polytropic solution of index $n$ so, apart from the outermost regions of the envelope, the density distribution can be approximated by (see Eq. (A.4) in Tylenda (2005) for $x \ll 1$ and $x_{0} \ll 1$ )

$\rho \simeq \rho_{0}\left(\frac{R_{0}}{r}\right)^{n}$,

where $\rho_{0}$ is the density at the base of the envelope at $r=R_{0}$. Then Eq. (B.3) can be rewritten as

$r^{n+1 / 2} \mathrm{~d} r=-\frac{\pi R_{2}^{2}}{M_{2}} \rho_{0} R_{0}^{n}\left(G M_{1}\right)^{1 / 2} \mathrm{~d} t$, which can be integrated giving

$$
r_{0}^{n+1 / 2}-r^{n+1 / 2}=\left(n+\frac{1}{2}\right) \frac{\pi R_{2}^{2}}{M_{2}} \rho_{0} R_{0}^{n}\left(G M_{1}\right)^{1 / 2} t,
$$

where $r_{0}$ is the radius of the orbit of the spiraling companion at $t=0$. Equation (B.6) can be used to obtain the time, $t_{\mathrm{s}}$, necessary for the comapnion to spiral from the initial orbit at $r_{0}$ to the base of the envelope at $r=R_{0}$, i.e.

$t_{\mathrm{s}}=\frac{t_{0}}{n+\frac{1}{2}}\left[\left(\frac{r_{0}}{R_{0}}\right)^{n+1 / 2}-1\right]$

where

$t_{0}=\frac{2}{3 \pi} \frac{R_{2}}{R_{0}} \frac{\rho_{2}}{\rho_{0}} P_{0}$,

where $P_{0}=2 \pi R_{0} \sqrt{R_{0} /\left(G M_{1}\right)}$ is the orbital period at the base of the envelope.

Equations (B.6) and (B.2) can be used to obtain the luminosity generated by the spiriling companion

$$
\begin{aligned}
L & =-\frac{\mathrm{d} E}{\mathrm{~d} t} \\
& =\frac{G M_{1} M_{2}}{2 R_{1} t_{0}}\left[\frac{t}{t_{\mathrm{s}}}+\left(1-\frac{t}{t_{\mathrm{s}}}\right)\left(\frac{r_{0}}{R_{0}}\right)^{n+1 / 2}\right]^{-\frac{n+3 / 2}{n+1 / 2}} .
\end{aligned}
$$




\section{References}

Armitage, P. J., \& Bonnell, I. A. 2002, MNRAS, 330, L11

Artymowicz, P., Clarke, C. J., Lubow, S. H., \& Pringle, J. E. 1991, ApJ, 370, L35

Bally, J., \& Zinnecker, H. 2005, AJ, 129, 2281

Banerjee, D. P. K., \& Ashok, N. M. 2004, ApJ, 604, L57

Banerjee, D. P. K., Varricatt, W. P., Ashok, N. M., \& Launila, O. 2003, ApJ, 598, L31

Banerjee, D. P. K., Varricatt, W. P., \& Ashok, N. M. 2004, ApJ, 615, L53

Blöcker, T., \& Schönberner, D. 1997, A\&A, 324, 991

Bode, M. F., O’Brien, T. J., \& Simpson, M. 2004, ApJ, 600, L63

Bond, H. E., Henden, A., Levay, Z. G., et al. 2003, Nature, 422, 405

Bond, H. E., \& Siegel, M. H. 2005, AJ, 131, 984

Brown, N. J. 2002, IAU Circ., 7785

Chabrier, G., \& Baraffe, I. 1997, A\&A, 327, 1039

Claussen, M., Healy, K., Starrfield, S., \& Bond, H. E. 2005, IAU Circ., 8602

Cohen, J. G. 1985, ApJ, 292, 90

Couderc, P. 1939, Ann. d'Astrophys., 2, 271

Cox, J. P., \& Giuli, R. T. 1984, Principles of Stellar Structure (New York: Gordon and Breach)

Crause, L. A., Lawson, W. A., Kilkenny, D., et al. 2003, MNRAS, 341,785

Deguchi, S., Matsunaga, N., \& Fukushi, H. 2005, PASJ, 57, L25

Desidera, S., \& Munari, U. 2002, IAU Circ., 7982

Drechsel, H., Rahe, J., Duerbeck, H. W., Kohoutek, H. W., \& Seitter, W. C. 1977, A\&AS, 30, 323

Frank, J., King, A., \& Reine, D. 2002, Accretion Power in Astrophysics, Third edition (Cambridge Univeristy Press)

Freitag, M., \& Benz, W. 2005, MNRAS, 358, 1133

Friedjung, M. 1992, A\&A, 262, 487

Goranskij, V. P., Shugarov, S. Yu., Barsukova, E. A., \& Kroll, P. 2004, IBVS, No. 5511

Han, Z., Podsiadlowski, Ph., \& Eggleton, P. P. 1995, MNRAS, 272, 800

Hartmann, L., \& Kenyon, S. J. 1996, ARA\&A, 34, 207

Hayashi, S. S., Yamamoto, M., \& Hirosawa, K. 1994, IAU Circ., 5942

Herwig, F. 2000, A\&A, 360, 952

Herwig, F. 2001, ApJ, 554, L71

Hessman, F. V. 1989, MNRAS, 239, 759

Iben, I. Jr. 1984, ApJ, 277, 333

Iben, I. Jr., \& Tutukov, A. V. 1992, ApJ, 389, 369

Kaminsky, B. M., \& Pavlenko, Y. V. 2005, MNRAS, 357, 38

Kato, T. 2003, A\&A, 399, 695

Kimeswenger, S. 2005, Astron. Nachr., 326, 657

Kimeswenger, S., Lederle, C., Schmeja, S., \& Armsdorfer, B. 2002, MNRAS, 336, L43
Kipper, T., Klochkova, V. G., Annuk, K., et al. 2004, A\&A, 416, 1107 Kolev, D., Mikołajewski, M., Tomov, T., et al. 2002, Collected Papers Physics (Shumen Univ. Press), 147

Lawlor, T. M. 2005, MNRAS, 361, 695

Lawlor, T. M., \& MacDonald, J. 2003, ApJ, 583, 913

Lombardi, J. C., Rasio, F. A., \& Shapiro, S. L. 1996, ApJ, 468, 797

Lombardi, J. C., Warren, J. S., Rasio, F. A., Sills, A., \& Warren, A. R. 2002, ApJ, 568, 939

Lombardi, J. C., Thrall, A. P., Deneva, J. S., Fleming, S. W., \& Grabowski, P. E. 2003, MNRAS, 345, 762

Lynch, D. K., Rudy, R. J., Russell, R. W., et al. 2004, ApJ, 607, 460

Martini, P., Wagner, R. M., Tomaney, A., et al. 1999, AJ, 118, 1034

Mould, J., Cohen, J., Graham, J. R., et al. 1990, ApJ, 353, L35

Munari, U., Desidera, S., \& Henden, A. 2002a, IAU Circ., 8005

Munari, U., Henden, A., Kiyota, S., et al. 2002b, A\&A, 389, L51

Munari, U., Henden, A., Vallenari, A., et al. 2005, A\&A, 434, 1107

Orio, M., Harbeck, D., Gallagher, J., \& Woodward, C. 2002, IAU Circ., 7892

Orio, M., Starrfield, S. G., \& Tepedenlegliolu, E. 2003, IAU Circ., 8110

Pontefract, M., \& Rawlings, J. M. C. 2004, MNRAS, 347, 1294

Podsiadlowski, Ph. 2003 [arXiv: astro-ph/0303660]

Rafanelli, P., \& Rosino, L. 1978, A\&AS, 31, 337

Retter, A., \& Marom, A. 2003, MNRAS, 345, L25

Rich, R. M., Mould, J., Picard, A., Frogel, J. A., \& Davies, R. 1989, ApJ, 341, L51

Ritchey, G. W. 1901, ApJ, 14, 293

Rushton, M. T., Coulson, I. M., Evans, A., et al. 2003, A\&A, 412, 767

Rushton, M. T., Geballe, T. R., Evans, A., et al. 2005a, MNRAS, 359, 624

Rushton, M. T., Geballe, T. R., Filippenko, A. V., et al. 2005b, MNRAS, 360, 1281

Soker, N., \& Tylenda, R. 2003, ApJ, 582, L105

Sparks, W. M., Starrfield, S., \& Truran, J. W. 1978, ApJ, 220, 1063

Stahler, S. W. 1994, PASP, 106, 337

Tylenda, R. 2004, A\&A, 414, 223

Tylenda, R. 2005, A\&A, 436, 1009

Tylenda, R., Crause, L., Górny, S. K., \& Schmidt, M. R. 2005a, A\&A, 439,651

Tylenda, R., Soker, N., \& Szczerba, R. 2005b, A\&A, 441, 1099

Vassiliadis, E., \& Wood, P. R. 1993, ApJ, 413, 641

Weidemann, V. 2000, A\&A, 363, 647

Wood, P. R., \& Zarro, D. M. 1981, ApJ, 247, 247

Yaron, O., Prialnik, D., Shara, M. M., \& Kovetz, A. 2005, ApJ, 623, 398

Young, P. A., Mamajek, E. E., Arnett, D., \& Liebert, J. 2001, ApJ, 556, 230 